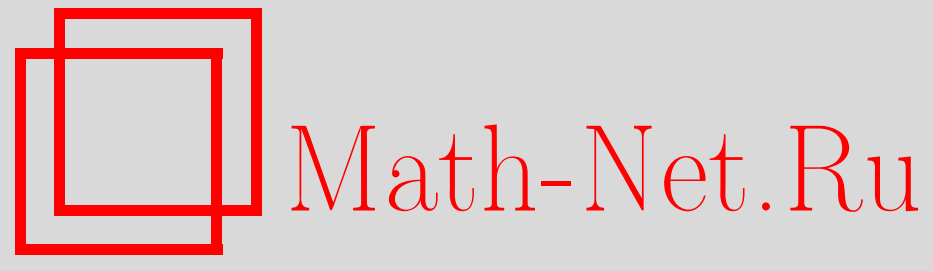

А. Ю. Хренников, Ш. Яамада, О концепции случайной последовательности относительно $p$-адических вероятностей, Теория вероятн. и ее примен., 2004, том 49, выпуск 1, 54-69

DOI: https://doi.org/10.4213/tvp236

Использование Общероссийского математического портала MathNet.Ru подразумевает, что вы прочитали и согласны с пользовательским соглашением

http://www . mathnet.ru/rus/agreement

Параметры загрузки:

IP : 54.196 .121 .252

26 апреля 2023 г., 16:55:43

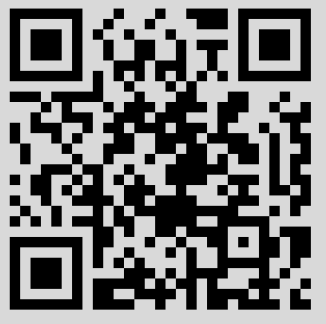




\section{О КОНЦЕПЦИИ СЛУЧАЙНОЙ ПОСЛЕДОВАТЕЛЬНОСТИ ОТНОСИТЕЛЬНО $p$-АДИЧЕСКИХ ВЕРОЯТНОСТЕЙ ${ }^{1)}$}

В статье продолжаются исследования по обобщенным вероятностным моделям, в которых вероятности принимают значения в полях $p$-адических чисел. Изучается $p$-адическое обобщение теории Мартин-Лёфа, основанной на тестах случайности. Использование такого обобщения является наиболее естественным подходом к $p$-адической случайности. Каждый тест случайности генерирует целую серию предельных теорем. Доказано, что можно эффективно перенумеровать все $p$-адические тесты случайности. Однако, в отличие от обычной теории Мартин-Лёфа для вещественных вероятностей, доказано, что не сушествует универсального теста случайности.

Ключевые слова и фразы: случайность, коллектив, модель Колмогорова, модель фон Мизеса, $p$-адические числа.

1. Введение. Обобщения вероятностных моделей, в которых вероятности принимают значения из поля $p$-адических чисел $[1],[2]$, были рассмотрены в рамках $p$-адических моделей в квантовой механике. С этим направлением можно ознакомиться в [3]-[10].

Применение $p$-адических чисел в физических и вероятностных исследованиях основывается на простом наблюдении, что поле рациональных чисел $\mathbf{Q}$ плотно не только в поле действительных чисел $\mathbf{R}$, но и в любом поле $p$-адических чисел $\mathbf{Q}_{p}$, где $p \geqslant 2$ - простое число. Таким образом, если мы начинаем построение модели с рациональных чисел, которые появляются в результате физических измерений (см. [5], [7]), то для анализа физических измерений можно использовать не только действительный анализ, но и $p$-адический. Более того, в силу теоремы Островского о вложении поля $\mathrm{Q}$ в поле с мультипликативной нормой, других полей просто не сушествует, см. п. 2. С другой стороны, $p$-адическая

* Department of Mathematics, Statistics and Computer Sciences, University of Växjö, S-35195, Sweden; e-mail: Andrei.Khrennikov@msi.vxu.se

** Department of Information Sciences, Science University of Tokio, Noda City, Chiba 278, Japan.

1) Работа выполнена при частичной поддержке гранта «Квантовая вероятность и проиложения к биологии и экономике» (Европейское Сообщество). 
вероятностная модель не эквивалентна колмогоровской вероятностной модели, и поэтому мы не можем использовать огромный потенциал колмогоровской модели для $p$-адических вероятностей, (см. [11]).

Первые $p$-адические модели [8] были попыткой расширить частотную конструкцию определения вероятностей, предложенную Р. фон Мизесом в теории «коллектива» [12], на $p$-адический случай, поскольку поведение относительных частот $\nu_{n}=n / N \in \mathrm{Q}$ можно изучать не только в $\mathbf{R}$, но и в $\mathbf{Q}_{p}$. Как известно, теория «коллектива» фон Мизеса основана на двух принципах: (1) принцип статистической стабилизации относительных частот и (2) принцип случайности, [12]. Это значит, что каждая бесконечная последовательность $(x)=\left(x_{1}, x_{2}, \ldots\right)$, где для простоты $x_{i}$ принимают значения из некоторого конечного множества $A$, называется «коллективом», если выполняются следующие условия:

1) частота $n(\alpha) / n$, где $n(\alpha)$ - число появлений элемента $\alpha$ среди первых $x_{1}, \ldots, x_{n}$, стремится к некоторому числу $p(\alpha)$ (вероятность $\alpha$ ) при $n \rightarrow \infty$ для любого $\alpha \in A$; это свойство последовательности $(x)$ называется принципом статистической стабилизации;

2) для $(x)_{\varphi}=\left(x_{\varphi(1)}, x_{\varphi(2)}, \ldots\right)$ - любсй выбранной подпоследовательности из $(x)$, где номера $\varphi(1), \varphi(2), \ldots$ не зависят от последовательности $(x)$, также выполняется принцип статистической стабилизации с теми же значениями вероятностей; это называется принципом случайности.

Первый принцип может быть естественным образом обобщен на $p$ адический случай, и $p$-адические вероятности определяются как пределы относительных частот в $p$-адической топологии. Однако, как и в обычной действительнозначной теории, тут также появляется трудность с принципом случайности. В p-адическом случае ситуация со стабилизацией пределов относительных частот в подпоследовательностях $(x)_{\varphi}$ даже хуже, чем в действительном случае, поскольку $p$-адическая метрика очень неустойчива: если $|n|_{p}=\varepsilon<1$, то $|n+1|_{p}=1$. В $p$-адическом случае мы не имеем даже возможности ограничить наши рассмотрения счетным числом подпоследовательностей $(x)_{\varphi}$, как, например, можно сделать в действительном случае (теорема Торнье [13]). Чтобы получить обоснованное определение $p$-адической случайности, мы использовали теорию колмогоровской сложности, см. [14]-[18]. Однако строгую математическую теорию развить не удалось (см. [7]), несмотря на достаточно интересные физические приложения [19], [20].

В этой работе мы изучаем $p$-адическое обобщение теории МартинЛёфа, [21], [22], основанное на тестах случайности. Такое обобщение выглядит как более естественный подход к $p$-адической случайности. Мы также даем естественные тесты для $p$-адичнозначных равномерных вероятностных распределений. Каждый такой тест индуцирует серию предельных теорем. С другой стороны, отдельная предельная теорема 
не является хорошим кандидатом, чтобы использовать ее как тест случайности, поскольку каждая такая теорема описывает только поведение подпоследовательности $S_{n_{k}}(\omega), S_{n}(\omega)=\xi_{1}(\omega)+\cdots+\xi_{n}(\omega)$, независимых одинаково распределенных случайных величин.

Показано, что возможно эффективно перенумеровать все $p$-адические тесты случайности. Однако, в отличие от теоремы Мартин-Лёфа для действительных вероятностей, доказано, что не существует универсального p-адического теста случайности.

В работе использована стандартная терминология книги М. Ли и П. Витани [18].

Первый автор хотел бы выразить благодарность Ю. В. Прохорову и В. М. Максимову за постоянный интерес к $p$-адическим моделям теории вероятностей.

2. $p$-адический анализ. Поля $p$-адических чисел $\mathbf{Q}_{p}$, где $p$ - простое число, были введены К. Гензелем в 1898 г. Первые 100 лет своего существования -p-адические числа использовались только в чистой математике. Но в последние годы эти числа были использованы также и в теоретической физике, см. [5].

Поле действительных чисел $\mathbf{R}$ строится как пополнение поля рациональных чисел $\mathbf{Q}$ относительно метрики $\rho(x, y)=|x-y|$, где $|\cdot|$ обычная метрика, заданная абсолютным значением. Поле $p$-адических чисел $\mathbf{Q}_{p}$ строится аналогичным образом, но с помощью других метрик. Для простого числа $p, p=2,3, \ldots, p$-адическая метрика $|\cdot|_{p}$ определяется следующим образом. Вначале определим ее для натуральных чисел. Так как каждое натуральное число $n$ можно единственным образом разложить в произведение простых чисел, $n=2^{r_{2}} \cdot 3^{r_{3}} \cdots p^{r_{p}}$, то можно положить $|n|_{p}=p^{-r_{p}}$ и $|-n|_{p}=|n|$. Тогда легко распространить определение $p$-адической метрики $|\cdot|_{p}$ на все рациональные числа, полагая $|n / m|_{p}=|n|_{p} /|m|_{p}$ для $m \neq 0$. Пополнение $\mathbf{Q}$ относительно метрики $\rho_{p}(x, y)=|x-y|_{p}$ есть локально компактное поле $p$-адических чисел $\mathbf{Q}_{p}$. Числовые поля $\mathbf{R}$ и $\mathbf{Q}_{p}$ являются единственными пополнениями поля рациональных чисел $\mathbf{Q}$, так как по теореме Островского [1], [2] метрики $|\cdot|,|\cdot|_{p}$, где $p$ - любое простое, являются единственно возможными метриками на $\mathbf{Q}$.

В отличие от вещественного расстояния, $p$-адическая метрика удовлетворяет строгому неравенству треугольника $|x+y|_{p} \leqslant \max \left(|x|_{p},|y|_{p}\right)$, $x, y \in \mathbf{Q}_{p}$. Обозначим $U_{r}(a)=\left\{x \in \mathbf{Q}_{p}:|x-a|_{p} \leqslant r\right\}$ и $U_{r}^{-}(a)=\{x \in$ $\left.\mathrm{Q}_{p}:|x-a|_{p}<r\right\}$, где $r=p^{n}$ и $n=0, \pm 1, \pm 2, \ldots$ Множества $U_{r}(a)$ и $U_{r}^{-}(a)$ являются замкнутыми и открытыми шарами в $\mathrm{Q}_{p}$, в то время как множество $\mathscr{S}_{r}(a)=\left\{x \in \mathrm{Q}_{p}:|x-a|_{p}=r\right\}$ является сферой в $\mathrm{Q}_{p}$ радиуса $r$. Эти множества (шары и сферы) имеют что-то странное в топологической структуре с точки зрения обычной евклидовой интуи- 
ции: они одновременно открыты и замкнуты. Другое интересное свойство $p$-адических шаров состоит в том, что они пересекаются тогда и только тогда, когда один из них содержится в другом. Также отметим, что каждая точка $p$-адического шара может быть взята как его центр. Таким образом, шар неоднозначно характеризуется своим центром и радиусом. Наконец, всякий $p$-адический шар $U_{r}(0)$ является аддитивной подгруппой $\mathbf{Q}_{p}$, в то время как шар $U_{1}(0)$ есть также кольцо, которое называется кольчом чельх $p$-адических чисел и обозначается $\mathbf{Z}_{p}$.

Каждое $x \in \mathbf{Q}_{p}$ имеет единственное каноническое разложение

$$
x=\frac{a_{-n}}{p^{n}}+\cdots+a_{0}+\cdots+a_{k} p^{k}+\cdots,
$$

которое сходится в норме $|\cdot|_{p}$ и где $a_{j} \in\{0,1, \ldots, p-1\}$ являются разрядами $p$-адического разложения. Элементы $x \in \mathbf{Z}_{p}$ имеют разложение $x=a_{0}+\cdots+a_{k} p^{k}+\cdots($ без отрицательных степеней $)$ и могут быть идентифицированы с последовательностями разрядов $x=\left(a_{0}, \ldots, a_{k}, \ldots\right)$, где $a_{j}$ принадлежат алфавиту $A_{p}=\{0,1, \ldots, p-1\}$.

Для любых $n, k \in \mathbf{N}$ обозначим $(n, k)$ наибольший общий делитель $n$ и $k$; для любого $n \in \mathbf{N}$ обозначим $M_{p}(n)$ остаток по модулю $p$ от $n, n=M_{p}(n) \bmod p$. Положим

$$
\Theta_{p}(n)= \begin{cases}\left|n-M_{p}(n)\right|_{p}, & n \geqslant p \\ 1, & 1 \leqslant n \leqslant p-1 .\end{cases}
$$

Следующий технический результат будет использован при построении $p$-адических рекурсивных тестов и в доказательстве предельных теорем для $p$-адических вероятностей.

Лемма 2.1. Пусть $n, k \in \mathbf{N}, p \leqslant k \leqslant n u M_{p}(n) \geqslant M_{p}(k)$. Тогда

$$
\left|\left(\begin{array}{l}
n \\
k
\end{array}\right)\right|_{p}=\frac{\Theta_{p}(n)}{\Theta_{p}(k)}
$$

Доказательст во. Пусть $n=\alpha+i p^{N}, k=\beta+j p^{l}$, где $i, j, N, l \in \mathbf{N}, 0 \leqslant \alpha, \beta \leqslant p-1$ и $(i, p)=(j, p)=1$. Тогда

$$
\left|\left(\begin{array}{l}
n \\
k
\end{array}\right)\right|_{p}=\left|\left(i p^{N}\right) \frac{\left(i p^{N}-p\right)}{p} \cdots \frac{\left(i p^{N}-j p^{l}+p\right)}{\left(j p^{l}-p\right)} \frac{1}{\left(j p^{l}\right)}\right|=\left|\frac{p^{N}}{p^{l}}\right|_{p}=p^{l-N} .
$$

Для получения (2) достаточно использовать соотношения $n-k+1=$ $i p^{N}-j p^{l}+(\alpha+1-\beta)$ и $0<\alpha+1-\beta \leqslant p$. Значит, последний член в знаменателе дроби $\left(\begin{array}{l}n \\ k\end{array}\right)=n \cdots(n-k+1) /(1 \cdots k)$, которой делится на $p$, равен $\left(i p^{N}-j p^{l}+p\right)$. Случаи, когда $n=\alpha$ или $k=\beta, 0 \leqslant \alpha, \beta \leqslant p-1$, рассматриваются аналогично. 
Лемма 2.2. Пусть $n, k \in \mathbf{N}, k \leqslant n, u M_{p}(n)+1 \leqslant M_{p}(k)$. Тогдa

$$
\left|\left(\begin{array}{l}
n \\
k
\end{array}\right)\right|_{p}=\Theta_{p}(n)
$$

Д ок а за т ел ьст в о. Пусть $n=\alpha+i p^{N}, k=\beta+j p^{l}$, где $(i, p)=$ $(j, p)=1,0 \leqslant \alpha, \beta \leqslant p-1$. Тогда

$$
\begin{aligned}
\left|\left(\begin{array}{l}
n \\
k
\end{array}\right)\right|_{p} & =\left|\left(i p^{N}\right) \frac{\left(i p^{N}-p\right)}{p} \frac{\left(i p^{N}-2 p\right)}{2 p} \cdots \frac{\left(i p^{N}-j p^{l}\right)}{\left(j p^{l}\right)}\right|_{p} \\
& =\left|p^{N}\right|_{p}=p^{-N}=\Theta_{p}(n) .
\end{aligned}
$$

Чтобы получить (4), достаточно заметить, что $n-k+1=\left(i p^{N}-j p^{l}\right)-$ $(\beta-\alpha-1)$ и $0 \leqslant \beta-\alpha-1<p$. Откуда последний член в знаменателе дроби $\left(\begin{array}{l}n \\ k\end{array}\right)=n \cdots(n-k+1) /(1 \cdots k)$, который делится на $p$, равен $i p^{N}-j p^{l}$. Случаи, когда $n=\alpha$ или $k=\beta, 0 \leqslant \alpha, \beta \leqslant p-1$, рассматриваются аналогично.

3. p-адические вероятностные меры на пространстве бинарных последовательностей. Положим $X=\{0,1\}, X^{n}=$ $\left\{\left(x_{1}, \ldots, x_{n}\right): x_{j} \in X\right\}, X^{*}=\bigcup_{n=0}^{\infty} X^{n}, X^{\infty}=\left\{\omega=\left(\omega_{1}, \ldots, \omega_{n}, \ldots\right)\right.$ : $\left.\omega_{j} \in X\right\}$. Для $x \in X^{n}$ положим $l(x)=n$. Для $x \in X^{*}, l(x)=n$, мы определим цилиндр $U_{x}$ с базисом $x: U_{x}=\left\{\omega \in X^{\infty}: \omega_{1}=x_{1}, \ldots, \omega_{n}=x_{n}\right\}$. Обозначим через $\mathscr{F}_{\text {суl }}$ алгебру подмножеств $X^{\infty}$, порожденную всеми цилиндрами.

Функция $\mathbf{P}: \mathscr{F}_{\text {суl }} \rightarrow \mathbf{Q}_{p}$ называется $p$-адической вероятностной мерой, если выполнены следуюшие условия: 1) аддитивность: $\mathbf{P}(A \cup B)=$ $\mathbf{P}(A)+\mathbf{P}(B), A \cap B=\varnothing ; 2)$ ограниченность: $\|\mathbf{P}\|_{p}=\sup \left\{|\mathbf{P}(A)|_{p}\right.$ : $\left.\left.A \in \mathscr{F}_{\text {суl }}\right\}<\infty ; 3\right)$ нормированность: $\mathbf{P}\left(X^{\infty}\right)=1$.

Тройка $\mathscr{P}=(\Omega, \mathscr{F}, \mathbf{P})$, где $\Omega=X^{\infty}, \mathscr{F}=\mathscr{F}_{\text {суl }}$, а $\mathbf{P}$ есть $p$-адическая вероятностная мера, будет называться $p$-адическим вероятностным пространством, см. [7] для обшего случая. В этой работе мы будем рассматривать равномерное -адическое распределение $\mathbf{P}_{p}: \mathscr{F}_{\text {cyl }} \rightarrow \mathbf{Q}_{p}$, для которого по определению $\mathbf{P}_{p}\left(U_{x}\right)=1 / 2^{l(x)}$ при $x \in X^{*}$. Заметим, что значения $\mathbf{P}_{p}$ на цилиндрах совпадают со значениями стандартного действительнозначного равномерного распределения $\mathbf{P}_{\infty}$, заданного на $X^{\infty}$. Так как $\mathbf{Q} \subset \mathbf{R}$ и $\mathbf{Q} \subset \mathbf{Q}_{p}$, то мы можем интерпретировать рациональные числа $1 / 2^{l(x)}$ и как действительные, и как $p$-адические.

Так как $\left|1 / 2^{l(x)}\right|_{2}=2^{l(x)}$, то $\mathbf{P}_{2}$ не является 2-адической вероятностной мерой. Поэтому мы ограничим наши рассмотрения случаем $p \neq 2$.

Отображение $j: X^{\infty} \rightarrow \mathbf{Z}_{2}, j(\omega)=\sum_{i=1}^{\infty} \omega_{i} 2^{i}$, дает взаимно однозначное соответствие между $X^{\infty}$ и $\mathbf{Z}_{2}$. Поэтому мы можем отождествить эти множества. Алгебра цилиндров $\mathscr{F}_{\text {суl }}$ совпадает с алгеброй $\mathscr{F}_{\mathrm{cl}}$ всех открыто-замкнутых подмножеств $\mathbf{Z}_{2}$, см. п. 2. Отождествление 
$X^{\infty}=\mathbf{Z}_{2}$ дает возможность использовать стандартную теорию интегрирования относительно $p$-адичнозначных мер для $p$-адических вероятностей. Различные варианты этой теории читатель может найти в [1], [2], [7]. Однако мы не будем использовать эту теорию в настоящей статье.

Функцию $f: X^{*} \rightarrow \mathrm{Q}_{p}$ мы будем называть рекурсивной, если найдется обычная рекурсивная функция $g: X^{*} \times \mathbf{N} \rightarrow \mathrm{Q}$ такая, что $\mid f(x)-$ $\left.g(x, k)\right|_{p}<1 / k . \quad p$-адическое распределение $\mathbf{P}$ будет называться рекурсивным, если функция $f_{p}: X^{*} \rightarrow \mathbf{Q}_{p}, f_{p}(x)=\mathbf{P}\left(U_{x}\right)$, рекурсивна.

В действительности в настоящей работе не будут рассматриваться общие рекурсивные $p$-адические распределения. Мы рассмотрим только одно равномерное $p$-адическое распределение $\mathbf{P}_{p}, p \neq 2$, которое, очевидно, является рекурсивным.

4. p-адические тесты случайности. Мы будем использовать следующие обозначения. Для каждого множества $M \subset X^{*}$ положим $M^{(n)}=\{x \in M: l(x)=n\}, n=1,2, \ldots$. Для каждого множества $W \subset X^{*} \times \mathbf{N}$ положим $W_{m}=\left\{x \in X^{*}:(x, m) \in W\right\}$. Откуда $W_{m}^{(n)}=\left\{x \in X^{*}: l(x)=n,(x, m) \in W\right\}$. Обозначим мощность конечного множества $A$ символом $\sigma(A)$. Мы не используем стандартный символ $|A|$, чтобы избежать выражения вида $\|A\|_{p}$.

Мы рассмотрим обобщение на случай $p$-адического равномерного распределения теста случайности, данного Мартин-Лёфом для обычных действительных вероятностей. Вначале дадим общее определение.

О п р е д е л е н и е 4.1. Пусть $\mathbf{P}$ - некоторое $p$-адическое рекурсивное распределение. Тогда рекурсивно перечислимое множество $V$, $V \subset X^{*} \times \mathbf{N}$, будет называться $p$-адическим $\mathbf{P}$-тестом случайности, если оно обладает следующими свойствами: для всех $n, m \in \mathbf{N}$

$$
\begin{gathered}
V_{m+1} \subset V_{m} \\
\left|\sum_{x \in V_{m}^{(n)}} \mathbf{P}\left(U_{x}\right)\right|_{p} \leqslant \frac{1}{p^{m}} .
\end{gathered}
$$

Мы используем $p$-адические тесты случайности для построения $p$-адических статистик. Пусть имеется выборочное пространство $X^{*}$ с ассоциированным $p$-адическим распределением $\mathbf{P}$. Для данного элемента $x$ выборочного пространства мы хотим проверить гипотезу « $x$ есть типичная выборка». Собственно говоря, свойство типичности $x$ есть свойство принадлежности к разумно определенному большинству. Чтобы удостовериться, принадлежит ли данный элемент выборочного пространства разумно выбранному большинству, мы используем понятие теста. Как и в обычной теории вероятностей, тест задается правилом, которое для каждого уровня значимости $\varepsilon=1 / p^{m}$ говорит нам, 
для каких элементов $x \in X^{*}$ гипотеза « $x$ принадлежит большинству $M$ в $X^{*} \gg$ должна быть отклонена, когда $\varepsilon=1-\mathbf{P}(M)$. Множество $V_{m}$ есть критическая область для уровня значимости $\varepsilon=1 / p^{m}$. Если $x \in V_{m}$, то гипотеза « $x$ принадлежит большинству $M$ в $X^{*} \gg$ отклоняется с уровнем значимости $\varepsilon$. Конечно, существует большая разница между «р-адическим большинством» и обычным «действительным большинством». Например, популяции, очень большие с точки зрения обычной вероятности, могут быть очень малы с точки зрения $p$-адической вероятности, и обратно.

В этой работе мы изучаем только равномерные $p$-адические распределения, где $\mathbf{P}=\mathbf{P}_{p}, p \neq 2$. Условимся тесты случайности для этих распределений называть просто $p$-адическими тестами. В нашем частном случае условие (6) может быть переформулировано в следующем виде:

$$
\left|\sigma\left(V_{m}^{(n)}\right)\right|_{p} \leqslant \frac{1}{p^{m}}
$$

(так как $\mathbf{P}\left(U_{x}\right)=1 / 2^{n}$ для $x \in V_{m}^{(n)}$ и $\left|2^{n}\right|_{p}=1$ для $p \neq 2$, неравенство (6) приобретает вид $\left.\left|\sum_{x \in V_{m}^{(n)}} 1\right|_{p} \leqslant 1 / p^{m}\right)$.

Предложение 4.1. Пусть $V$ есть р-адический тест. Тогда для каждого $(x, m) \in V$ имеет место неравенство

$$
l(x)\left(\log _{p} 2\right)>m \geqslant 1 .
$$

Доказ а т ельст т о. Пусть $n=l(x)$. Так как $x \in V_{m}$, мы имеем $V_{m}^{(n)} \neq \varnothing$, и в силу (7) число $\sigma\left(V_{m}^{(n)}\right)$ делится на $p^{m}$. Таким образом, $2^{n}=\sigma\left(X^{n}\right) \geqslant \sigma\left(V_{m}^{(n)}\right) \geqslant p^{m}$, что доказывает неравенство (8).

Предложение 4.2. Пусть $V$ есть $p$-адический тест. Тогда для любых $k \geqslant m, n \in \mathbf{N}$ имеет место неравенство

$$
\left|\sigma\left(V_{m}^{(n)} \backslash V_{k}^{(n)}\right)\right|_{p} \leqslant \frac{1}{p^{m}} .
$$

Д о к а з а т е л ь с т в о. Так как $V_{k}^{(n)} \subset V_{m}^{(n)}$, то

$$
\sigma\left(V_{m}^{(n)}\right)=\sigma\left(V_{k}^{(n)}\right)+\sigma\left(V_{m}^{(n)} \backslash V_{k}^{n}\right) .
$$

В силу строгого неравенства треугольника мы получим

$$
\left|\sigma\left(V_{m}^{(n)} \backslash V_{k}^{(n)}\right)\right|_{p} \leqslant \max \left(\left|\sigma\left(V_{m}^{(n)}\right)\right|_{p},\left|\sigma\left(V_{k}^{(n)}\right)\right|_{p}\right)=\frac{1}{p^{m}} .
$$

Как обычно, обозначим целую часть действительного числа $x$ через $[x]$. Условие (8) может быть переписано в виде

$$
\left[l(x) \log _{p} 2\right] \geqslant m .
$$


Функция $\lambda(n)=\left[n \log _{p} 2\right], n \in \mathbf{N}$, будет играть важную роль в наших рассмотрениях. Для всякого $p$-адического теста $V$ и $n \in \mathbf{N}$ только множества $V_{m}^{(n)}, m=1, \ldots, \lambda(n)$, могут быть непусты.

Дадим несколько примеров $p$-адических тестов случайности. Bсе эти тесты связаны с поведением сумм

$$
S(x)=x_{1}+\cdots+x_{n}, \quad x \in X^{*}, \quad n=l(x) .
$$

П р и м е р 4.1. Положим

$$
\begin{gathered}
V_{m}=\left\{x \in X^{*}: \Theta_{p}(S(x)) \geqslant p^{m} \Theta_{p}(l(x)), S(x) \neq 0, l(x) \geqslant p\right. \\
\text { и } \left.M_{p}(S(x)) \leqslant M_{p}(l(x))\right\} .
\end{gathered}
$$

Итак, мы рассматриваем последовательности из нулей и единиц длины, большей, чем $p$, отличные от чисто нулевых последовательностей. Условие $M_{p}(S(x)) \leqslant M_{p}(l(x))$ имеет следуюций смысл: остаток по модулю $p$ суммы $S(x)$ не больше, чем остаток по модулю $p$ длины последовательности испытаний. Условие $\Theta_{p}(S(x)) \geqslant p^{m} \Theta_{p}(l(x))$ имеет следующий смысл: пусть число испытаний за вычетом его остатка по модулю $p$ точно делится на $p^{s}$, и пусть сумма $S(x)$ без ее остатка по модулю $p$ точно делится на $p^{t}$. Тогда $m$ не превосходит $s-t$.

Покажем, что множество $V=\left\{(x, m): x \in V_{m}\right\}$ есть $p$-адический тест. Для этого достаточно доказать справедливость (7). Имеем

$$
\sigma\left(V_{m}^{(n)}\right)=\sum_{k}\left(\begin{array}{l}
n \\
k
\end{array}\right)
$$

где $p \leqslant k \leqslant n$ и $M_{p}(k) \leqslant M_{p}(n), \Theta_{p}(n) / \Theta_{p}(k) \leqslant 1 / p^{m}$. Чтобы получить $(7)$, достаточно использовать строгое неравенство треугольника и лемму 2.1 .

П р и м е р 4.2. Положим

$$
\bar{V}_{m}=\left\{x \in X^{*}: \Theta_{p}(l(x)) \leqslant \frac{1}{p^{m}}, M_{p}(S(x)) \geqslant M_{p}(l(x))+1\right\} .
$$

Используя лемму 2.2 , получим, что (7) справедливо для $\bar{V}_{m}$. Таким образом, множество $\bar{V}=\left\{(x, m): x \in \bar{V}_{m}\right\}$ является $p$-адическим тестом.

П р и м е р 4.3 (конечные тесты). Пусть $n \in \mathbf{N}$ - фиксированное число, а $T$ - некоторое подмножество $X^{n},|\sigma(T)|_{p}=p^{-\lambda(n)}$. Положим $W_{m}^{(n)}=T$ для $m=1, \ldots, \lambda(n)$ и $V_{j}^{(n)}=\varnothing, j>\lambda(n), V_{j}^{(n)}=\varnothing, k \neq n$, для всех $j=1,2, \ldots$ Тогда $V=\left\{(x, m): x \in V_{m}\right\}, V_{m}=\bigcup_{k=1}^{\infty} V_{m}^{(k)}$, есть конечный $p$-адический тест.

Чтобы проиллюстрировать тесты (12) и (13), полезно рассмотреть некоторые им соответствующие подмножества с фиксированными значениями $M_{p}(n)$ и $M_{p}(S(x))$. 
Начнем с теста (12). Положим

$$
\begin{aligned}
V_{m}(1,0) & =\left\{x \in V_{m}: M_{p}(l(x))=1, M_{p}(S(x))=0\right\} \\
V(1,0) & =\left\{(x, m): x \in V_{m}(1,0)\right\}
\end{aligned}
$$

Этот тест связан с выборками вида

$$
x=\left(x_{1}, \ldots, x_{1+j p^{N}}\right), \quad N \in \mathbf{N}, \quad(j, p)=1 .
$$

Такая выборка должна быть отклонена с уровнем значимости $\varepsilon=1 / p^{m}$, если $1>|S(x)|_{p} \geqslant p^{m}|l(x)-1|_{p}=p^{m-N}$. Таким образом, тест $V(1,0)$ отклоняет все выборки вида $x=\left(x_{1}, \ldots, x_{1+j p^{N}}\right),(j, p)=1$, в которых сумма $S(x)=x_{1}+\cdots+x_{1+j p^{N}}$ не делится на достаточно высокую степень $p$, но делится на $p^{1}$.

Выборка $x$ вида (15), для которой $S(x)=i p^{k},(i, p)=1, k \geqslant 1$, отвергается с уровнем значимости $\varepsilon=1 / p^{m}$, если $k<N-n$.

Для теста $(12)$ и $M_{p}(l(x))=1$ можно фиксировать $M_{p}(S(x))=1$ и получить новый тест:

$$
\begin{aligned}
V_{m}(1,1) & =\left\{x \in V_{m}: M_{p}(l(x))=1, M_{p}(S(x))=1\right\} \\
V(1,1) & =\left\{(x, m): x \in V_{m}(1,1)\right\}
\end{aligned}
$$

Тогда выборка $x$ вида (15) должна быть отвергнута с уровнем значимости $\varepsilon=1 / p^{m}$, если $1>|S(x)-1|_{p} \geqslant p^{m}|l(x)-1|_{p}=p^{n-N}$. Таким образом, тест $V(1,1)$ бракует все выборки $x$ вида $(15)$, для которых $S(x)-1$ не делится на достаточно высокую степень $p$, но делится на $p^{1}$.

Таким же образом, фиксируя $M_{p}(n)=s=0,1, \ldots, p-1$, мы получим тесты $V_{m}(s, q), q=0,1, \ldots, s$. Тест $V(s, q)$ отвергает некоторые выборки вида

$$
x=\left(x_{1}, \ldots, x_{s+j p^{N}}\right), \quad j, N \in \mathbf{N}, \quad(j, p)=1,
$$

а именно выборки $x$, для которых число $S(x)-q$ не делится на достаточно высокую степень $p$, но делится на $p^{1}$. Выборка $x$ вида $(17)$, для которой $S(x)=q+i p^{k},(i, p)=1, k \geqslant 1$, отвергается с уровнем значимости $\varepsilon=1 / p^{m}$, если $k<N-m$.

Изучим теперь тест (13). Условие $M_{p}(S(x)) \geqslant M_{p}(l(x))+1 \geqslant 0$ означает, что этот тест используется, чтобы отвергнуть некоторые выборки, для которых сумма $S(x)$ не делится на $p$ (cp. с (14)). Положим

$$
\begin{aligned}
\bar{V}_{m}(0,1) & =\left\{x \in \bar{V}_{m}: M_{p}(l(x))=0, M_{p}(S(x))=1\right\}, \\
\bar{V}(0,1) & =\left\{(x, m): x \in \bar{V}_{m}(0,1)\right\} .
\end{aligned}
$$

Этим тестом мы отбрасываем с уровнем значимости $\varepsilon=1 / p^{m}$ все выборки вида $x=\left(x_{1}, \ldots, x_{j p^{N}}\right),(j, p)=1$, для которых $N<m$ 
и $M_{p}(S(x))=1$. Можно сравнивать тесты $\bar{V}(0,1)$ и $V(0,0)$. Последний тест используется для отбраковки выборок того же вида, но для которых сумма $S(x)$ делится на $p: S(x)=i p^{k},(i, p)=1, k \geqslant 1$. Выборка отклоняется с уровнем значимости $\varepsilon=1 / p^{m}$, если $k<N-m$.

Можно ввести $p$-адический тест $O$, который покрывает все случаи делимости на $p$ суммы $S(x)$.

Предложение 4.3. Пусть $\Phi u \Psi-\partial в а$ р-адических теста, для которых $\Phi \cap \Psi=\varnothing$. Тогда множество $\Gamma=\Phi \cup \Psi$ есть $p$-адический тест с критической областью $\Gamma_{m}=\Phi_{m} \cup \Psi_{m}$ и с уровнем значимости $\varepsilon=1 / p^{m}$.

Д ок а з а т е л ь с т в о. Нам нужно только доказать, что (7) справедливо. Но $\left|\sigma\left(\Gamma_{m}^{(n)}\right)\right|_{p}=\left|\sigma\left(\Phi_{m}^{(n)}\right)+\sigma\left(\Psi_{m}^{(n)}\right)\right|_{p} \leqslant \max \left(\left|\sigma\left(\Phi_{m}^{(n)}\right)\right|_{p}\right.$, $\left.\left|\sigma\left(\Psi_{m}^{(n)}\right)\right|_{p}\right) \leqslant 1 / p^{m}$.

Возвратимся к тестам $V$ и $\bar{V}$, определенным в примерах 4.1 и 4.2 . Очевидно, что $V_{m} \cap \bar{V}_{m}=\varnothing$ для всех $m$. Тогда множества $\Sigma_{m}=V_{m} \cup \bar{V}_{m}$ дают критические области $p$-адического теста $\Sigma=\left\{(x, m): x \in \Sigma_{m}\right\}$.

5. Некоторые предельные теоремы. Как в обычной теории вероятностей, тесты $V$ и $\bar{V}$ примеров $4.1,4.2$ связаны с некоторыми предельными теоремами $p$-адической теории вероятностей. Пусть $\mathscr{P}=$ $\left(\Omega, \mathscr{F}_{\text {cyl }}, \mathbf{P}\right)$ - вероятностное пространство, образованное равномерным

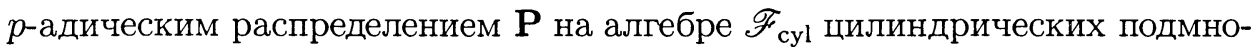
жеств $\Omega=X^{\infty}, p \neq 2$. Для $\omega \in \Omega$ положим $S_{n}(\omega)=\omega_{1}+\cdots+\omega_{n}$.

Теорема 5.1. Для каждого $l \in \mathbf{N}$

$$
\mathbf{P}\left\{\omega \in \Omega:\left|S_{n}(\omega)-M_{p}\left(S_{n}(\omega)\right)\right|_{p}=\frac{1}{p^{l}}, M_{p}\left(S_{n}(\omega)\right) \leqslant M_{p}(n)\right\} \longrightarrow 0
$$

в $\mathrm{Q}_{p}$, когда $\left|n-M_{p}(n)\right|_{p} \rightarrow 0$.

Д о к а з а т е л с т в о. Используя условия примера 4.1, получаем, что

$$
\begin{aligned}
& \mathbf{P}\left\{\omega \in \Omega:\left|S_{n}(\omega)-M_{p}\left(S_{n}(\omega)\right)\right|_{p}=\frac{1}{p^{l}}, M_{p}\left(S_{n}(\omega)\right) \leqslant M_{p}(n)\right\} \\
& \quad \leqslant p^{l}\left|n-M_{p}(n)\right|_{p} .
\end{aligned}
$$

В частности, мы получаем следующие предельные теоремы.

Следствие 5.1 (см. [22]). Для каждого $l \in \mathbf{N}$ вероятности

$$
\mathbf{P}\left\{\omega \in \Omega: S_{n}(\omega) \in \mathscr{S}_{1 / p^{l}}(0)\right\} \quad u \quad \mathbf{P}\left\{\omega \in \Omega: S_{n}(\omega) \in \mathscr{S}_{1 / p^{l}}(1)\right\}
$$

стремятся $к$ нулю в $\mathrm{Q}_{p}$, когда $|n-1|_{p}$ стремится $\kappa$ нулю.

Следствие 5.2. Для каждого $l \in \mathbf{N}$ вероятности $\mathbf{P}\{\omega \in \Omega$ : $\left.S_{n}(\omega) \in \mathscr{S}_{1 / p^{l}}(0)\right\}$ стремятся $\kappa$ нулю в $\mathrm{Q}_{p}$, когда $|n|_{p} \rightarrow 0$. 
Формально, следствие 5.1 можно интерпретировать следующим образом. Сумма $S_{n}(\omega)$ может быть рассмотрена как сумма $S_{n}(\omega)=$ $\xi_{1}(\omega)+\cdots+\xi_{n}(\omega)$ независимых одинаково распределенных случайных величин $\xi_{j}(\omega)$, принимающих значения 0 или 1 с вероятностями $\frac{1}{2}$ (см. [7]). По следствию 5.1 распределение вероятностей случайной величины $S_{\lim }(\omega)=\lim _{n \rightarrow 1} S_{n}(\omega)$ сконцентрировано в точках $a_{0}=0$ и $a_{1}=1$ из $\mathbf{Q}_{p}$. В силу симметрии $\mathbf{P}_{S_{\mathrm{lim}}}\left(\left\{a_{0}\right\}\right)=\mathbf{P}_{S_{\mathrm{lim}}}\left(\left\{a_{1}\right\}\right)=\frac{1}{2}$. Конечно, это есть только формальное утверждение, поскольку следствие 5.1 дает сходимость только на сферах $\mathbf{Q}_{p}$.

Теорема 5.2. Имеет место сходимость в $\mathrm{Q}_{p}$ :

$$
\mathbf{P}\left\{\omega \in \Omega: M_{p}\left(S_{n}(\omega)\right) \geqslant M_{p}(n)+1\right\} \longrightarrow 0,
$$

когда $\left|n-M_{p}(n)\right|_{p} \rightarrow 0$.

Как и в случае теоремы 5.1, мы можем, например, положить $M_{p}(n)=0$ или $M_{p}(n)=1$ и получить следующие следствия теоремы 5.2.

Следствие 5.3. Вероятности $\mathbf{P}\left\{\omega \in \Omega: M_{p}\left(S_{n}(\omega)\right) \geqslant 1\right\}$ cmpeмятся к нулю в $\mathbf{Q}_{p}$, когда $|n|_{p} \rightarrow 0$.

Следствие 5.4. Вероятности $\mathbf{P}\left\{\omega \in \Omega: M_{p}\left(S_{n}(\omega)\right) \geqslant 2\right\}$ cmpeмятся к нулю в $\mathbf{Q}_{p}$, когда $|n-1|_{p} \rightarrow 0$.

Заметим, что

$$
\left.\mathbf{P}\left\{\omega \in \Omega: M_{p}\left(S_{n}(\omega)\right) \geqslant 1\right)\right\}=\mathbf{P}\left\{\omega \in \Omega: S_{n}(\omega) \in \mathscr{S}_{1}(0)\right\}
$$

Таким образом, из следствий 5.2 и 5.3 мы получим, что

$$
\mathbf{P}\left\{\omega \in \Omega: S_{n}(\omega) \in U_{1 / p^{m}}(0)\right\} \longrightarrow 1
$$

в $\mathbf{Q}_{p}$, когда $|n|_{p} \rightarrow 0$, для всех $m \in \mathbf{N}$. Отсюда формально получаем, что вероятностное распределение $\mathbf{P}_{S_{\lim }}$ величины $S_{\lim }=\lim _{n \rightarrow 0} S_{n}(\omega)$ сконцентрировано в точке $a_{0}=0 \in \mathbf{Q}_{p}: \mathbf{P}_{S_{\text {lim }}}(\{0\})=1$.

По видимому, в $p$-адическом случае более естественно использовать тесты случайности, чем предельные теоремы. В противоположность обычной теории вероятностей, в $p$-адическом случае нет общих предельных теорем для $n \rightarrow \infty$. Все предельные теоремы дают сходимость .вероятностей для некоторых подпоследовательностей $n_{k} \rightarrow \infty, k \rightarrow \infty$. Например, из $\left|n_{k}\right|_{p} \rightarrow 0, n_{k} \neq 0$, вытекает, что $n_{k}=j p^{N},(j, p)=1$, $N \rightarrow \infty$, а из $n_{k} \neq 1,\left|n_{k}-1\right|_{p} \rightarrow 0$ вытекает, что $n_{k}=1+j p^{N},(j, p)=1$, $N \rightarrow \infty$, и т.д.

6. Рекурсивное перечисление множества $p$-адических тестов. Здесь мы докажем, что множество всех $p$-адических тестов рекурсивно перечислимо. Общая схема доказательства такая же, как и в классическом случае. Тем не менее, основная часть доказательства - 
построение алгоритма $p$-адического теста на основе частично рекурсивной функции - принципиально иная. Вначале напомним одну хорошо известную лемму (см., например, [18]).

Лемма 6.1. Существует частично рекурсивная функция $f: \mathbf{N} \times$ $\mathbf{N} \rightarrow X^{*} \times N$ со следуюшими свойствами:

(a1) если $i, j \in \mathbf{N} u f(i, j) \neq \infty$, mо $f(i, k) \neq \infty$ для всех $k \leqslant j$;

(а2) множество $A \subset X^{*} \times N$ рекурсивно перечислимо тогда $u$ только тогда, когда $A=\{f(i, j): j=1,2, \ldots\} \backslash\{\infty\}$ для некоторого $i \geqslant 1$.

О п р ед ле н и е 6.1. Для всякого теста $V$ положим $V_{m}=$ $\{x:(x, m) \in V\}$ и $V_{m}^{(n)}=\left\{x: x \in V_{m} \wedge l(x)=n\right\}$.

Теорема 6.1. Множество всех р-адических тестов рекурсивно перечислимо.

Д ок а з а т ль с т в о. Пусть задана частично рекурсивная функция $\Phi: \mathbf{N} \rightarrow X^{*} \times \mathbf{N}$ такая, что если $\Phi(n) \downarrow$, то $\Phi(k) \downarrow$ для $1 \leqslant k<n$. Идея доказательства состоит в конструировании из такой функции $\Phi$ общей рекурсивной функции $g: \mathbf{N} \rightarrow X^{*} \times \mathbf{N}$, для которой $A_{g}=$ range $g$ есть $p$-адический тест.

3 а м е ч а н и е 6.1. Если данная $\Phi$ уже является $p$-адическим тестом, то $A_{g}=$ range $g=$ range $\Phi$. Условие, что range $g$ и range $\Phi$ равны, всегда выполняется в силу конструкции $\Phi$. Таким образом, только то условие, что range $g$ является $p$-адическим тестом, осталось непроверенным.

Тест $T$, равный range $g$ по определению, конструируется шаг за шагом, с использованием следующего алгоритма. Заметим, что при этом на каждом шаге получаем $p$-адический тест.

Обозначения. Список $\mathscr{D}_{m}^{(n)}$ используется, чтобы дать аппроксимацию для множества $T_{m}^{(n)}$ в процессе вычислений. Список $\mathscr{R}_{m}^{(n)}$ используется, чтобы собирать элементы $x$ из $A_{\Phi}=\operatorname{range} \Phi$ такие, что $l(x)=n \wedge(x, m) \in \operatorname{range} \Phi$.

Основная идея состоит в том, что мы не можем увеличивать множества $\mathscr{D}_{m}^{(n)}$ на шаге, когда $\Phi(j)=(x, m) \wedge l(x)=n$, из-за того, что $p$-адическая метрика очень чувствительна к изменению количества единиц.

В список $\mathscr{R}_{m}^{(n)}$ элементы собираются до тех пор, пока не будет $k p^{m}$ элементов.

После этого мы изменяем $\mathscr{D}_{m}^{(n)}$, полагая $\mathscr{D}_{m}^{(n)}=\mathscr{R}_{m}^{(n)}$.

Так как мы желаем получить множество, которое обладает свойством $T_{m} \supset T_{m+1}$, мы должны проверить условие $\mathscr{D}_{m-1}^{(n)} \supset \mathscr{R}_{m}^{(n)}$, прежде чем положить $\mathscr{D}_{m}^{(n)}=\mathscr{R}_{m}^{(n)}$.

С другой стороны, из возрастания $\mathscr{D}_{m}^{(n)}$ на некотором шаге может вытекать, что множество $\mathscr{R}_{m+1}^{(n)}$, сконструированное на предыдущих шагах, имеет $s p^{m}$ элементов и станет меньше, чем $\mathscr{D}_{m}^{(n)}=\mathscr{R}_{m}^{(n)}$. 
В этом случае мы положим $\mathscr{D}_{m+1}^{(n)}=\mathscr{R}_{m+1}^{(n)}$ и продолжим проверять это условие, увеличивая $m+2, m+3, \ldots<\left(\log _{p} 2\right) n$.

Чтобы $T$ было рекурсивно перечислимым множеством, мы построим общую рекурсивную функцию $g: \mathbf{N} \rightarrow X^{*} \times \mathbf{N}$ с $T$, paвным range $g$ по определению. (Ясно, что если range $\Phi$ уже есть $p$-адический тест, то $T=\operatorname{range} \Phi$, но это не ясно в общем случае.)

Ниже мы будем использовать обозначения, принятые в языке Паскаль, для построения нашего алгоритма.

\section{Алгоритм.}

3 а м е ч а н и е 6.2. Мы используем две таблицы LOOP $j$ и BAR для переходов и идентификатор valuei, чтобы контролировать текущее значение $i$.

$\operatorname{begin} T:=\varnothing ;$ Set $\mathscr{D}_{m}^{(n)}=\mathscr{R}_{m}^{(n)}=\varnothing$ for $1 \leqslant m \leqslant\left[n \log _{p} 2\right]$;

$$
j:=0 ; i:=1 ; t_{m}^{(n)}:=0 ;
$$

LOOP $j$ : for $j=1$ to $\infty$ do

begin if $\Phi(j) \downarrow$ then

\{else if $\Phi(j) \uparrow$, computation of $T$ is finished

$$
\begin{aligned}
& \operatorname{begin}(x, m):=\Phi(j) ; n=l(x) ; \\
& \quad \text { if } m>\left[n \log _{p} 2\right] \text { then begin } T:=\varnothing ; \text { halt end; } \\
& \qquad \mathscr{R}_{m}^{(n)}:=\mathscr{R}_{m}^{(n)} \cup\{x\} ; \\
& \quad t_{m}^{(n)}:=t_{m}^{(n)}+1 ; \\
& \quad \text { if }\left|t_{m}^{(n)}\right|_{p} \leqslant \frac{1}{p^{m}} \text { and } \mathscr{D}_{m-1}^{(n)} \supset \mathscr{R}_{m}^{(n)} \text { then } \\
& \qquad \mathscr{D}_{m}^{(n)}:=\mathscr{R}_{m}^{(n)} \\
& \text { end; }\left\{\text { end of } \operatorname{loop}_{\text {on }} j\right\} \\
& g:=\left(\text { foo }\left(i, m, \mathscr{D}_{m}^{(n)}\right)\right)_{1} ; \\
& \text { valuei }:=\left(\text { foo }\left(i, m, \mathscr{D}_{m}^{(n)}\right)\right)_{2} ; s:=m ;
\end{aligned}
$$

BAR: $s:=s+1$

$$
\begin{aligned}
& \text { if } s \leqslant\left[n \log _{p} 2\right] \text { and }\left|t_{1}^{(n)}\right|_{p} \leqslant \frac{1}{p^{m}} \text { and } \mathscr{D}_{s-1}^{(n)} \supset \mathscr{R}_{s}^{(n)} \text { then } \\
& \text { begin } \mathscr{D}_{s}^{(n)}:=\mathscr{R}_{s}^{(n)} ; g:=\left(\text { foo }\left(\text { valuei, } s, \mathscr{D}_{s}^{(n)}\right)\right)_{1} ; \\
& \text { goto BAR }
\end{aligned}
$$

end;

$$
T:=T \bigcup_{m \leqslant s<\left(\log _{p} 2\right) n}\left\{\mathscr{D}_{s}^{(n)} \times\{s\}\right\} ;
$$

goto LOOP;

end. $\{$ end of program $\}$ 
З а м е ч а н и е 6.3. Функциональная программа foо принимает набор $(i, m, \mathscr{D})$ как вход и возвращает foo $(i)$, которое является парой $(g$,valuei $)$, где $g$ есть список $\left(\left(i,\left((\mathscr{D})_{1}, m\right)\right),\left(i+1,\left((\mathscr{D})_{2}, m\right)\right), \ldots\right.$, $\left.\left(i+l(\mathscr{D})-1,\left((\mathscr{D})_{l(\mathscr{D})}, m\right)\right)\right)$, и valuei есть текущее значение $i+l(\mathscr{D})$ переменной $i$.

Здесь проекция на $i$-й элемент списка $x$ записана как $(x)_{i}$

$$
\begin{aligned}
& \text { function foo }(i, m \text { : integer, } \mathscr{D} \text { : list }) \text { : list; } \\
& \text { var } g \text { : list; } \\
& \text { var } x \text { : integer; } \\
& \text { begin } g:=\text { nil; } x:=0 ; \\
& \text { while } \mathscr{D} \neq \text { nil do } \\
& \qquad \begin{aligned}
\text { begin } g:=g \cup\left\{(i+x),\left((\mathscr{D})_{x}, m\right)\right\} ; \\
\quad i:=i+1 ; x:=x+1 \\
\text { end }
\end{aligned} \\
& \text { foo }:=(g, i+x-1)
\end{aligned}
$$

end.

$T$ по построению всегда будет $p$-адическим тестом для равномерного распределения. В частности, множество $T$ рекурсивно перечислимо, так как $T$ есть range $g$ с общей рекурсивной функцией $g$.

\section{7. О несуществовании $p$-адического универсального теста.} Рассмотрим естественное обобщение определения универсального теста случайности.

О п р е д е л е н и е 7.1. Некоторый $p$-адический тест $U$ называется универсальным, если для каждого $p$-адического теста $V$ мы можем эффективно найти $c \in \mathbf{N}$ (зависящее от $U$ и $V$ ) такое, что $V_{m+c} \subset U_{m}$ для всех $m$.

Хорошо известно, что в обычной теории вероятностей существует универсальный тест случайности, который, конечно, не является единственным. Однако в $p$-адической теории вероятностей не существует универсального рекурсивного теста.

Мы начнем с технических рассмотрений. Рассмотрим более аккуратно свойства функции $\lambda(n)=\left[n \log _{p} 2\right]$. Если $p>2$, то мы имеем $\log _{p} 2<1$. Положим $L_{k}=\left[k / \log _{p} 2\right]$. Если $0<n \leqslant L_{1}$, то $n \log _{p} 2<1$ и $\lambda(n)=0$. С другой стороны, если $L_{k-1}<n \leqslant L_{k}$, то $\lambda(n)=k-1, k \geqslant 2$. Положим $n_{k}=L_{k}+1$.

Лемма 7.1. Неравенство

$$
p^{\lambda\left(n_{k}\right)}>2^{n_{k}-1}
$$

справедливо для всех $k=1,2, \ldots$ 
Д ок азате льст в о. Мы имеем $\lambda\left(n_{k}\right)=k$ и $\lambda\left(n_{k}-1\right)=k-1$. По определению $\lambda(n)=\max \left\{l: p^{l}<2^{n}\right\}$. Следовательно, для всех $n$ имеем $p^{\lambda(n)+1}>2^{n}$. В частности, $p^{\lambda\left(n_{k}-1\right)+1}=p^{k}>2^{n_{k}-1}$, откуда $p^{k}=$ $p^{\lambda\left(n_{k}\right)}>2^{n_{k}-1}$.

Построим теперь два $p$-адических теста $W$ и $\widetilde{W}$, используя следующую процедуру.

Для $k=1,2, \ldots$ и $j=1, \ldots, \lambda\left(n_{k}\right)$ положим

$$
\begin{aligned}
& W_{j}^{\left(n_{k}\right)}=W_{\lambda\left(n_{k}\right)}^{\left(n_{k}\right)}=\left\{x_{1}, \ldots, x_{p^{\lambda\left(n_{k}\right)}}\right\}, \\
& \widetilde{W}_{j}^{\left(n_{k}\right)}=\widetilde{W}_{\lambda\left(n_{k}\right)}^{\left(n_{k}\right)}=\left\{x_{2^{n_{k}-p^{\lambda\left(n_{k}\right)}+1}}, \ldots, x_{2^{n_{k}}}\right\}
\end{aligned}
$$

и $W_{l}^{\left(n_{k}\right)}=\widetilde{W}_{l}^{\left(n_{k}\right)}=\varnothing$ для $n \neq n_{k}$ и $l=1,2, \ldots$. Здесь мы использовали лексикографическую нумерацию элементов $X^{n_{k}}, k=1,2, \ldots$ : $x_{1}, x_{2}, \ldots, x_{2^{n_{k}}}$. Так как $\sigma\left(W_{j}^{\left(n_{k}\right)}\right)=\sigma\left(\widetilde{W}_{j}^{\left(n_{k}\right)}\right)=p^{\lambda\left(n_{k}\right)}, j=1, \ldots, \lambda(n)$, то в силу (20) мы получим $W_{j}^{\left(n_{k}\right)} \cap \widetilde{W}_{j}^{\left(n_{k}\right)} \neq \varnothing$, откуда

$$
X^{n_{k}}=W_{j}^{\left(n_{k}\right)} \cup \widetilde{W}_{j}^{\left(n_{k}\right)}, \quad j=1, \ldots, \lambda\left(n_{k}\right) .
$$

Теорема 7.1. Не существует универсального р-адического теcma.

Д ок а за т е л ь с т в. Допустим, что такой универсальный $p$-адический тест $U$ существует. Тогда можно эффективно найти $c_{1}, c_{2} \in \mathbf{N}$ такие, что $W_{m+c_{1}} \subset U_{m}$ и $\widetilde{W}_{m+c_{2}} \subset U_{m}$, где $W$ и $\widetilde{W}-p$-адические тесты, построенные выше. Пусть $k$ выбрано достаточно большим, чтобы вєполнялись неравенства $\lambda\left(n_{k}\right)-c_{1} \geqslant 1$ и $\lambda\left(n_{k}\right)-c_{2} \geqslant 1$. Тогда $W_{1+c_{1}}^{\left(n_{k}\right)}=W_{\lambda\left(n_{k}\right)}^{\left(n_{k}\right)}$ и $\widetilde{W}_{1+c_{2}}^{\left(n_{k}\right)}=\widetilde{W}_{\lambda\left(n_{k}\right)}^{\left(n_{k}\right)}$. Откуда

$$
U_{1}^{\left(n_{k}\right)} \supset W_{1+c_{1}}^{\left(n_{k}\right)} \cup \widetilde{W}_{1+c_{2}}^{\left(n_{k}\right)}=X^{n_{k}} .
$$

Из этого вытекает

$$
\left|\sigma\left(U_{1}^{\left(n_{k}\right)}\right)\right|_{p}=\left|\sigma\left(X^{n_{k}}\right)\right|_{p}=1,
$$

что противоречит (7).

\section{СПИСОК ЛИТЕРАТУРЫ}

1. Schikof W. Ultrametric Calculus. Cambridge: Cambridge Univ. Press, 1984, 306 p.

2. Koblitz N. p-Adic Analysis. A Short Course on Recent Work. Cambridge: Cambridge Univ. Press, 1980, $163 \mathrm{p}$.

3. Volovich I. V. p-adic string. - Classical Quantum Gravity, 1987, v. 4, p. 83-87.

4. Freund P. G. O., Witten E. Adelic string amplitudes. - Phys. Lett. B, 1987, v. 199, № 2, p. 191-194. 
5. Владимиров В. С., Волович И. В., Зеленов Е.И. р-адический анализ и математическая физика. М.: Наука, 1994, 352 с.

6. Frampton P. H., Okada Y. p-adic string $N$-point function. - Phys. Rev. Lett. B, 1988 , v. 60, № 6, p. 484-486.

7. Khrennikov A. Yu. p-Adic Valued Distributions in Mathematical Physics. Dordrecht: Kluwer, 1994, $264 \mathrm{p}$.

8. Хренников $A$. Ю. -адическая теория вероятностей и ее приложения. Принцип статистической стабилизации частот. - Теорет. матем. физика, 1993, т. 97, № 3, c. 348-363.

9. Хренников А.Ю. О вероятностных распределениях на поле $p$-адических чисел. Теория вероятн. и ее примен., 1995, т. 40, в. 1, с. 189-192.

10. Хренников А.Ю. О расширении частотного подхода Р. фон Мизеса и аксиоматического подхода А.Н. Колмогорова на $p$-адическую теорию вероятностей. Теория вероятн. и ее примен., 1995, т. 40, в. 2, с. 458-464.

11. Колмогоров A.H. Основные понятия теории вероятностей. М.-Л.: ОНТИ, 1936, $80 \mathrm{c}$.

12. von Mises $R$. Mathematical Theory of Probability and Statistics. New York-London: Academic Press, 1964, 694 p.

13. Tornier E. Wahrscheinlichkeitsrechnunug und Allgemeine Integrationstheorie. Leipzig: Teubner, 1936, $160 \mathrm{p}$.

14. Solomonoff $R$.J. A formal theory of inductive inference. I, II. - Information and Control, 1964, v. 7, p. 1-22, 224-254.

15. Колмогоров $A . H$. Три подхода к определению понятия «количество информации». - Проблемы передачи информации, 1965, т. 1, № 1, с. 3-11.

16. Uspensky V.A., Shen A. Relations between varieties of Kolmogorov complexities. Math. Systems Theory, 1996, v.29, № 3, p. 271-292.

17. Звонкин A.K., Левин Л. А. Сложность конечных объектов и обоснование понятий информации и случайности с помощью теории алгоритмов. - Успехи матем. наук, 1970, т. 25 , № 6 , с. 85-127.

18. Li M., Vitányi P. An Introduction to Kolmogorov Complexity and Its Applications. New York: Springer-Verlag, 1997, 637 p.

19. Хренников А.Ю. Описание экспериментов по нахождению $p$-адической статистики в квантовых дифракционных экспериментах. - Докл. РАН, 1998, т. 58, № 3, c. 478-480.

20. Khrennikov A. Yu. p-adic probability predictions of correlations between particles in the two slit and neuron interferometry experiments. - Nuovo Cimento Soc. Ital. Fis. B (12), 1998, v. 113, №6, p. 751-760.

21. Мартин-Лё П. О понятии случайной последовательности. - Теория вероятн. и ее примен., 1966, т. 11, в. 1, с. 198-200.

22. Martin-Löf $P$. The definition of random sequences. - Information and Control, 1966, v. 9, p. $602-619$.

23. Хренников $A$. Ю. $p$-адическая асимптотика бернуллиевских вероятностей. - Теория вероятн. и ее примен., 1997, т. 42 , № 4 , с. 839-845.

Поступила в редакцию 28.VI. 2000 